\section{Clinical Case Reports: Striking the Right Balance}

DOI: 10.3766/jaaa.31.1.1 $\mathrm{s}$ both a clinician and an editor, one of the types of journal submissions I am always excited to send out for review is the clinical case report. In the medical literature, the clinical case report has always served an important role. This includes presenting to the reader a case that is rare or one that offers a particular set of findings that have not been previously reported.

In all areas of medicine, there has been a notable decline in the number of clinical case reports published (Nissen and Wynn, 2014). As research methods in the medical field advanced and expanded in the latter half of the 20th century, enthusiasm for case reports diminished and has been replaced by quantitative evidence-based research studies (Nissen and Wynn, 2012).

This has been further magnified by the pressure to perform well on the current metrics that academic journals use to determine the quality of their manuscripts. These metrics include the impact factor, Eigenfactor, $h$-index, and immediacy index, to name a few. Most of these measures have some type of relationship to the number of times articles are cited.

Due to the fact that most clinical case reports are retrospective and consist of uncontrolled observations of symptoms, comorbities, and treatments, they are referenced less often than controlled hypothesis-driven experiments. Regardless of this fact, it has been argued by proponents that the clinical case report allows for clinical awareness, is highly educational, and may act to help providers identify similar patients. I wholeheartedly agree with this notion.

As editors of the Journal of the American Academy of Audiology (JAAA), we consistently work to provide content that is of interest to our readers. JAAA is the official journal of the American Academy of Audiology (the Academy), an organization where 8 percent of the membership has earned a $\mathrm{PhD}$ that would be considered a research-oriented degree.

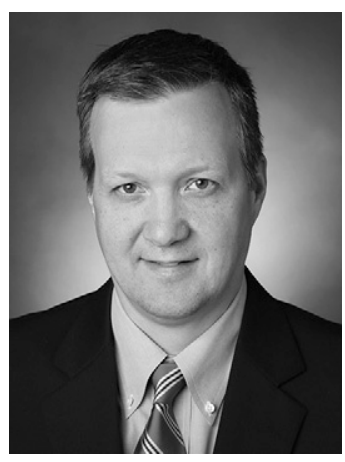

Of course, there are AuDs and master's-level audiologists who are primarily involved in research and, likewise, $\mathrm{PhDs}$ who are involved primarily in clinic or administrative activities. The important point, however, is that the lion's share of the membership of the organization that JAAA serves is composed of practicing clinicians.

At the Academy's annual convention, the grand rounds presentations that represent the oral analog of clinical case reports are always some of the most well-attended sessions. When the manuscript submissions for this year (so far) are tabulated, however, only 8 percent of the submissions are clinical case reports that are directly relevant to the practicing clinical audiologist. Is this the right balance?

There is no question that there is a high degree of interest in the heavily research-oriented content in JAAA. When the full-text downloads of articles are analyzed, JAAA consistently ranks in the top 100 for the number of downloads out of more than 11,000 titles (at Number 19 in September 2019), according to the most recent report from IngentaConnect, the JAAA online content host.

And, speaking of balance, in this issue of the journal we have a clinical case report focused on dizziness and hearing loss. Dizziness is extremely common, although it has many forms, some that are notoriously difficult to diagnosis and treat. The case report in this issue is titled "Symptoms, Audiometric and Vestibular Laboratory Findings, and Imaging in a Concurrent Superior Canal Dehiscence Syndrome and Vestibular Schwannoma."

In this report, Garrison and colleagues describe the complex presentation of two rare clinical entities occurring at the same time. The write-up includes an exhaustive case history and detailed images of the diagnostic findings, as well as treatments provided. I encourage you to read it-and to submit your own.

Although there are limitations to case reports, such as bias and the inability to provide epidemiological 
information, it is my opinion that the educational merits and potential to change clinical practice far outweigh these issues.

Devin L. McCaslin, PhD Deputy Editor-in-Chief

\section{REFERENCES}

Nissen T, Wynn R. (2012) The recent history of the clinical case report: a narrative review. JRSM Short Rep 3(12):87.

Nissen T, Wynn R. (2014) The history of the case report: a selective review. JRSM Open 5(4):2054270414523410. 